\title{
50 \\ Two-layer MIMO Network Coding based Scheme for Cooperative Communications
}

\author{
Hung Viet Nguyen, Dam Viet Nguyen and Sy Quy Nguyen* \\ Posts and Telecommunications Institute of Technology, Hanoi, Vietnam
Emails: \{hungnv_vt1, damnv, synq\}@ptit.edu.vn
}

\begin{abstract}
In this contribution, we propose and investigate a novel scheme of two-layer multi-input multi-output (MIMO) systems employing network coding in the scenario of cooperative communication. The first MIMO layer of our system is constituted from an Irregular Convolutional Coded Unity Rate Coded Space Time Trellis Coded M-ary Phase Shift Keying (IrCC-URC-STTC-MPSK) schemes. The MIMO coding scheme is designed with the aid of Extrinsic Information Transfer (EXIT) charts for approaching the corresponding channel capacity in fast fading environment. The second Network Coding based Virtual-MIMO layer is incorporated into our system for coping with the bad effect of slow fading channels, which may not be handled by the first MIMO coding layer. Based on simulation results, our proposed scheme outperforms the conventional MIMO systems, where no cooperation is invoked for exploiting the diversity gain brought by the aiding of other users available in the system.
\end{abstract}

Keywords - Cooperative Communications, Network Coding, MIMO.

\section{INTRODUCTION}

The ultimate aim of designing a wireless communication system is to provide reliable high data rate links, which can be improved by utilizing various types of diversity techniques, e.g. spatial-diversity, temporal-diversity and frequency-diversity. The socalled cooperative diversity, a form of spatial diversity, has been introduced in [1], [2] in order to create a Virtual Antenna Array (VAA) with the aid of cooperating single-antenna-aided mobiles for providing independent fading transmission paths.

Space Time Trellis Codes (STTCs) [3] and Space Time Block Codes (STBCs) [4], which are joint coding and transmit-receive diversity aided MIMO systems, constitute efficient techniques of communicating over fading channels [5]. We note that STTCs are capable of attaining coding gain in addition to their spatial diversity gain, while the
STBCs of [6] can only achieve a spatial diversity gain [7] but no coding gain. Additionally, Tuchler and Hagenauer [8], [9] proposed the employment of IRregular Convolutional Codes (IRCCs)for serially concatenated schemes, which are constituted by a family of convolutional codes having different rates, in order to design a near-capacity system. They were specifically designed with the aid of Extrinsic Information Transfer (EXIT) charts conceived for analyzing the convergence properties of iterative decoding aided concatenated coding schemes [10]. As a further advance, it was shown in [11],[12] that a recursive Unity-Rate Code (URC) having an infinite impulse response is capable of efficiently spreading the available extrinsic information across the entire iterative receiver chain. This URC may be employed as an intermediate code, in order to improve the attainable decoding convergence. The URC may be viewed as a precoder invoked for creating a serially concatenated inner coding component having an Infinite Impulse Response (IIR) in order to reach the $(1,1)$ point in the EXIT chart and hence to achieve an infinitesimally low Bit Error Ratio (BER) [13], as detailed in [7]. As a result, IrCC-URC-STTC coding arrangement may be employed for the sake of approaching the Discrete-input Continuous-output Memoryless Channel (DCMC) capacity of the MIMO systems.

The cooperative diversity protocols proposed in $[1,2]$ typically rely on two main phases: direct transmission phase (broadcast phase) and relaying phase (cooperative phase). Based on the behaviour of relays, the cooperative diversity aided systems fall into four main categories, namely Amplify and Forward (AF), Decode and Forward (DF), hybrid AF/DF [14] and coded cooperation [15]. As the epitome of collaboration, network coding is a recently 
Research, Development and Application on Information and Communication Technology

introduced paradigm [16] conceived for efficiently disseminating data in multicast wireless networks, where the data flows arriving from multiple sources are combined to achieve compression and hence to increase the achievable throughput, as well as to reduce the delay imposed and to enhance the errorresilience [17]. The basie principle of network coding [16] is that nodes in the network form combinations of messages that they receive, and forward those combinations; the destination then recovers the original messages from different combinations it receives.

Network codes may be classified based on different perspectives. For example on the basis of how the information streams are processed at the relays [18], or depending on the construction of network codes [19], depending on the specific the architecture of networks employing network coding [20], the layer in networks where the network coding operates [21], just to name a few. Three well-known categories of network codes are Linear Network Codes (LNC), Non-linear Network Codes (NLNC) and the family of so-called Hybrid Network Codes(HNC). It is suggested by [22], [23] that LNC has many attractive properties. From a theoretical standpoint, linearity is a beneficial algebraic property supported by exact mathematical foundations. From an engineering standpoint, the simplicity of linear approaches leads to relatively low complexity in the encoding and decoding processes. In LNC systems, the relay nodes store the incoming packets in their own buffer and then transmit the linear combinations of these packets. The coefficients used for creating the linear combination may be random numbers defined over a large finite field [24], [25], or those gleaned from parity-check matrices of error control codes [26], [27].

Generalized Dynamic Network Codes (GDNC) constitute an extension of the Dynamic Network Codes (DNC) recently proposed in [28]. Further designs were provided in [27], [29] by considering the problem as being equivalent to that of designing linear block codes defined over $G F(q)$ for erasure correction. In [27], [29], the authors investigated the GDNC system assuming an idealised or so-called "perfect" channel coding scheme, which was defined as the code that is capable of operating at the Continuous-input Continuous-output Memoryless Channel's (CCMC) capacity. Some of recent work has extended the network coding scheme to the scenario of employing realistic Single-Input Single-Ouput (SISO) channel coding schemes [30], [31].

Against this background, the novel contribution of this paper is that the two-layer MIMO systems are proposed and designed for combating negative effects of both the shadow fading and of the small-scale (fast) Rayleigh fading in hostile wireless channels. First, a realistic near capacity MIMO channel coding scheme, considered as the first MIMO layer, is designed for the sake of supporting cooperative multi-user communications employing network codes in smallscale fading environment. Second, the linear network coding layer, considered as a virtual MIMO layer, is added on the top of the first MIMO layer for providing a good performance of the system in appearance of severe large-scale(slow) fading in the wireless channels. The design guidelines presented in this contribution can also be extended to a diverse range of two-layer MIMO systems employing network coding in the scenario of cooperative communications. The rest of this paper is organized as follows.

In Section II, our proposed system model is illustrated, before providing details of our design principles as well as design examples for the first MIMO layer and the second virtual-MIMO (VMIMO) layer of our system in Sec. III and Sec. IV, respectively. In Section V, we present our simulation results and associated discussions, before offering our conclusions.

\section{SYSTEM MODEL}

In this paper, we consider a class of the two-layer MIMO multi-user cooperative systems having the system model portrayed in Fig.1,where $M$ users cooperatively communicate with a common base station (BS). A transmission session is conducted in two groups of phases, the broadcast phases and the cooperative phases. During the broadcast phases, each user broadcasts $k_{1}$ of its own information frames, namely $I_{m}(t), t=[m, m+$ $\left.m, \ldots,\left(k_{1}-1\right) M+m\right]$, where $I_{m}(t)$ in Fig. 1 represents an information frame (packet or message) transmitted by User $m, m \in\{1,2 \ldots, \mathrm{M}\}$, which takes place during the specific 
broadcast phase $t$ from the whole set of $k_{1} M$ broadcast phases. Then, during the cooperative phases, each user transmits $k_{2}$ parity frames, namely $\boxplus m\left(t^{\prime}\right), t=[m, M+$ $\left.m, \ldots,\left(k_{2}-1\right) M+m\right]$, containing nonbinary linear combinations of its own information frames and of the information frames transmitted by the $(M-1)$ other users. The notation $\boxplus m\left(t^{\prime}\right)$ in Fig.1 denotes the parity frame transmitted by User $m$ during the cooperative phase $k_{2}(m-1)+t^{\prime}$, and representsthe specific nonbinary linear combination, where the weighting coefficients will be defined imminently in context of Fig.1. Note that a single phase is defined as a time period, in which a user performs a single transmission, and the transmissions are conducted by $\mathrm{M}$ users via the appropriately created orthogonal channels either in the time, frequency or code domain. We consider orthogonal channels in the time domain.

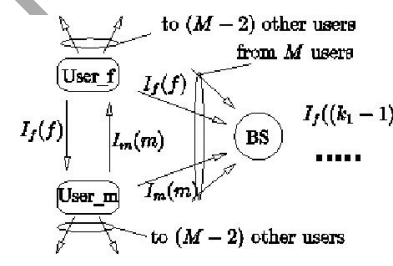

$\left(B_{1}\right)$ Broaricast phases $m$ and $f$

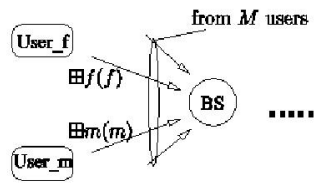

$\left\langle C_{1}\right\rangle$ Cooperative phasea $m$ and $f$
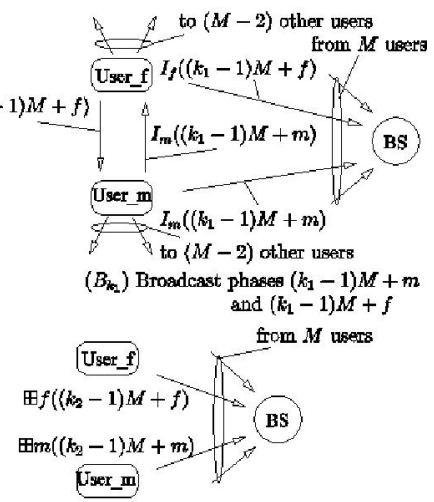
$\left(C_{k_{2}}\right)$ Cooperative phases $\left(k_{2}-1\right) M+m$
and $\left(k_{2}-1\right\rangle M+f$

Fig.1. General system model of the two layer MIMO system.

Our system may be deemed as consisting of two layers, where the first layer MIMO layer characterizes the point-topoint transmission in the system, as detailed in Sec.II-A. The second layer VMIMO layer represents how signals from different users are processed for forming a VMIMO system, which is further described in Sec. II-B.

\section{A. The first MIMO layer structure}

In the first MIMO layer structure portrayed in Fig.2, the bit stream at the transmitter side is first encoded by the IrCC encoder before it is fed into the interleaver $\pi_{1}$, in order to get the interleaved input stream of the URC encoder. The output of the URC encoder is again scrambled by the interleaver $\pi_{2}$ before being encoded by the STTC. This signal is then transmitted to the receiver side over wireless fading channels, as portrayed in Fig.2.
At the receiver side, as seen in Fig. 2, the signals provided by the receive antenna are demodulated and decoded by the STTC decoder before being processed by the $I$ inner iterations exchanging extrinsic information between the STTC decoder and the URC decoder. The resultant soft information extracted from the received signals by the inner iterations are then used as the input data for the $J$ outer iterations exchanging extrinsic information between the IrCC encoder and the amalgamated inner component, which is the Unity Rate Coded M-ary Phase Shift Keying (URC-STTC-MPSK) arrangement. Hence, our design goals presented in Sec. III are to determine the most appropriate number of inner and outer iterations, as well as the weighting coefficients of the IrCC subcodes used in the IrCC encoder.

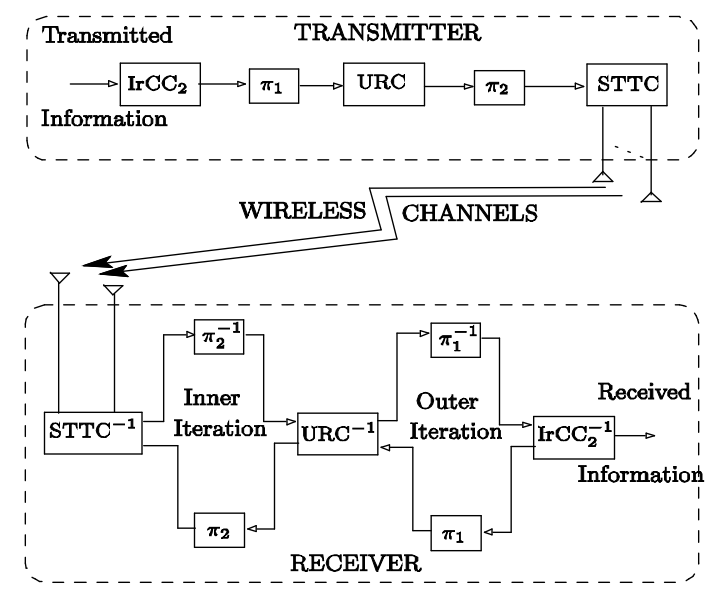

Fig.2. Structure of the Irregular Convolutional Coded Unity Rate Coded Space Time Trellis Code (IrCCURC-STTC) coding scheme.

\section{B. The second Network-Coding based VMIMO layer}

The second layer of our system represent how signals received from different users are processed, in order to construct VMIMO-like effects. The signal process pictured in Fig. 2 can be conveniently illustrated by the transfer matrix shown in Fig.3, where $P_{m s}(t)$ corresponds tothe parity coefficient for the information frame $I_{m}(t)$ contained in the $s^{\text {th }}$ parity frame transmitted by User mduring the cooperative phase $k_{2}(m-1)+s$. Note that the column $k_{2}(m-1)+s$ of the parity matrix $P_{k_{1} M \times k_{2} M}$ shown in Fig. 3 is the set of the parity coefficients valid for the nonbinary linear combination, which forms the ${ }^{\text {th }}$ parity frame transmitted by User $m, s \in\{1, \ldots k\}$, during the cooperativephase $k_{2}(m-1)+s$.

Following the approach presented in [27] and [29], the system's transfer matrix illustrated in Fig. 3 can be derived 
Research, Development and Application on Information and Communication Technology

from the appropriately selected systematic generator matrix $\mathrm{G}$ of an $\left(n, k, d_{m i n}\right.$ linear block code, which is shown in Fig.4. The linear block code employed should be a Maximum Distance Separable (MDS) code for the sake of achieving the minimum distance bound [32]. In this paper, we use Reed Solomon (RS) codes, which constitute a wellknown class of MDS codes. The systematic generator matrix G of RS codes defined over $G F(q)$ is provided by the software application SAGE [33]. We follow [28] and assume that the BS knows how each parity frame was constructed. Naturally, the related side-information imposes an additional overhead, albeit this may be deemed negligible, when a sufficiently long information frame is used.

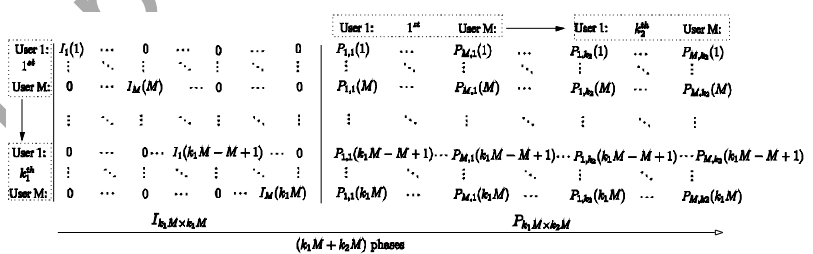

Fig.3. Transfer matrix illustrating the transmission session of the system having $M$ users transmitting in

$$
\begin{array}{cccccccc}
\multicolumn{10}{c}{\left(k_{1} M+k_{2} M\right)} & \text { phases. } \\
G_{k \times n} & & & \\
1 & 0 & \cdots & 0 & P_{1,1} & P_{1,2} & \cdots & P_{1, n-k} \\
0 & 0 & \cdots & 0 & P_{1,1} & P_{1,2} & \cdots & P_{1, n-k} \\
\vdots & \vdots & \ddots & \vdots & \vdots & \vdots & \ddots & \vdots \\
0 & 0 & \cdots & 1 & P_{1,1} & P_{1,2} & \cdots & P_{1, n-k} \\
& I_{k \times k} & & & P_{k \times n-k}
\end{array}
$$

Fig.4. Generator matrix $G_{k \times n}$ used to form the transfer matrix.

\section{THE FIRST MIMO LAYER DESIGN}

In this section, we first summarize the formula used for calculating the channel capacity of the MIMO system, in order to provide benchmarks for our design and corresponding results presented in subsequent sections of Sec. III-B and Sec. III-C, respectively.

\section{A. Capacity MIMO Systems}

Let us consider a single transmission link associated with the transmitted and received signals of $x$ and $y$, respectively. The received signal can be represented as

$$
y=h x+n
$$

where $\mathrm{h}=\mathrm{h}_{\mathrm{s}} \mathrm{h}_{\mathrm{f}}$ is the complex-valued fading coefficient that comprises two components, a slow fading coefficient (large-scale shadow fading or quasistatic fading) $h_{s}$, which is constant for all symbols within a frame and a fast fading (small-scale Rayleigh fading) coefficient $h_{f}$, which varies on a symbol by symbol basis, while $\mathrm{n}$ is the Additive White Gaussian Noise (AWGN) process having a variance of $\mathrm{N}_{0} / 2$ per dimension.

We refer to $\mathrm{C}$ as the maximum achievable transmission rate of reliable communication supported by this channel. Let us assume that the transmitter encodes data at a rate of $\mathrm{R}$ bits $/ \mathrm{s} / \mathrm{Hz}$. If the channel realisation $\mathrm{h}$ has a capacity of $\left.\mathrm{C}\right|_{\mathrm{h}}<R$, which is lower than required, thesystem is declared to be in outage, where the outage probability is given by

$$
P_{e}(R)=\operatorname{Pr}\left\{\left.C\right|_{h}<R\right\}
$$

with $\left.\mathrm{C}\right|_{h}$ being the capacity, i.e. the maximum achievable rate of the channel, when $h$ is known. If $x$ is an independent and identically distributed (i.i.d.) random variable, the transmission link obeys the CCMC model. A similar approach may be applied to MIMO system, where its the capacity was shown to be [34]

$$
C^{D C M C}(\eta)=\eta-\frac{1}{2^{\eta}} \sum_{l=1}^{l=2^{\eta}} E\left[\log _{2} \sum_{z=1}^{z=2^{\eta}} \exp \left(\psi_{l, z}\right) \mid X_{l}\right]
$$

where $L=2^{\eta}$ is the number of modulation levels, and $E\left[A \mid X_{1}\right]$ is the expectation of $A$ conditioned on the L-ary signals $X_{l}$, whereas $\psi_{1, z}$ is given by

$$
\psi_{l, z}=\frac{-\left|h\left(x_{l}-x_{z}\right)+n_{l}\right|^{2}+\left|n_{z}\right|^{2}}{N_{0}}
$$

\section{B. EXIT-chart matching and optimization of the $\mathrm{IrCC}$ - URC-STTC-MPSK scheme}

This section is dedicated to the design of our first coding layer employing the IrCC-URC-STTC-MPSK coding arrangement portrayed in Fig. 2 and detailed in Sec.II-A. Accordingly, our main design goals are to determine the most appropriate number of inner and outer iterations, as well as the weighting coefficients of the IrCC subcodes used in the IrCC encoder. We present the design for the IrCC-URC-STTC-QPSK 
arrangement employing 17-subcode IrCC as a numerical example.

Firstly, we design the two stage inner arrangement URC-STTC-QPSK. Secondly, the code design is continued by viewing our three-stage IrCC-URCSTTC-QPSK coding arrangement as the two-stageconcatenated IrCC outer code and the amalgamated URC-STTC-QPSK inner code. The details of our design using the EXIT chart matching procedure conceived for this coding arrangement is briefly summarized as follows:

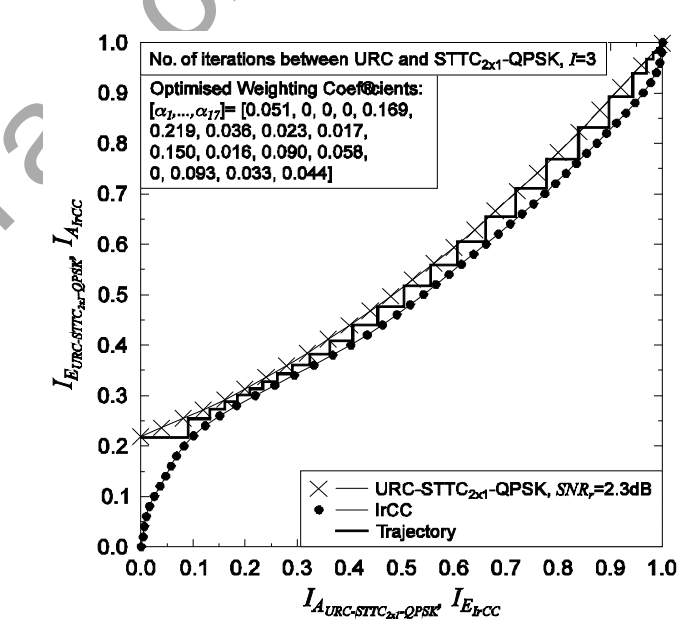

Fig.5. The EXIT chart curves of the URC-STTCQPSK having two transmit and one receive antennas (URC-STTC2 $\times 1-Q P S K)$ scheme at SNR $=2.3 \mathrm{~dB}$ when $I=3$ terations between URC and STTC $2 \times 1$ QPSK are configured and of the IrCC having the overall coding rate $R_{c}=0.5$.

Step1.1: In order to increase the achievable channel capacity of the amalgamated URC-STTCQPSK inner code and to approach that of a STTC and STTC-QPSK aided system, an iterative decoding process exchanging extrinsic information between the URC and STTC decoders should be implemented [35]. We exploit the above-mentioned characteristics of EXIT charts [36] for calculating the DCMC capacity of the two inner-most coding arrangements, namely that of the STTC-QPSK and URC-STTCQPSK schemes. Then, based on the capacity of these two coding arrangements, we determine the most appropriate number of iterations. As a result, Fig.6 shows that once at least $I=3$ iterations had been applied, the achievable channel capacities of the
STTC-QPSK and URC-STTC-QPSK systems become near-identical.

Step1.2: Create the EXIT chart of the URC-STTCQPSK scheme for different receiver Signal to Noise Ratios $R_{r}$, as seen in Fig.5.

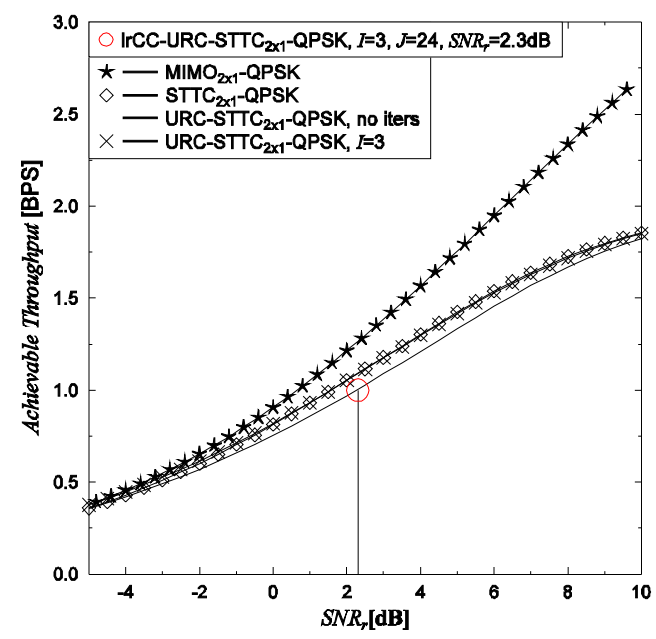

Fig. 6. Achievable throughputs of the MIMO2 $\times 1$ QPSK, the STTC $2 \times 1-Q P S K$ arrangement, the URC$S T T C 2 \times 1-Q P S K$ arrangement $(I=0: 3$ iterations between URC and URC-STTC $2 \times 1-Q P S K)$ and the IrCC-URC-STTC $2 \times 1-Q P S K$ arrangement $(I=3$ iterations between URC and URC-STTC $2 \times 1-Q P S K$ in conjunction with $\mathrm{J}=24$ iterations between IrCC and URC-STTC2 $\times 1-Q P S K)$.

Step 2: Fix the overall IrCC code rate to $R_{C}=0.5$ and employ the EXIT curve matching algorithm [8] for generating the optimisedweighting coefficients $\alpha_{j}, j=1, \ldots, 17$, of the 17 -subcode IrCC codes corresponding to the lowest possible $S N R_{r}$ that allows decoding convergence, where the decoding trajectory reaches the top-right corner of the corresponding EXIT charts. This observation suggests that a nearcapacity performance can be achieved.

Again, by exploiting the area property of the EXIT-charts [36], [37], the achievable DCMC capacities of the $\mathrm{MIMO}_{2 \times 1}-\mathrm{QPSK}$, the $\mathrm{STTC}_{2 \times 1}$ QPSK, URC-STTC $2 \times 1$-QPSK and IrCC-URCSTTC $_{2 \times 1}$-QPSK aided systems are quantified by generating the EXIT charts of these schemes across various SNR values. Then, the areas under each of these EXIT charts corresponding to different $S N R$ 
Research, Development and Application on Information and Communication Technology

values are measured for determining the associated capacity curves, as plotted in Fig. 6.

It should be noted that the capacity of an inner arrangement sets an upper bound for the capacity of an outer arrangement. Hence, according to the aforelisted order, the capacity associated with the inner most arrangement $\mathrm{MIMO}_{2 \times 1}$-QPSK sets the maximum achievable capacity for all the systems employing the other schemes, namely the $\mathrm{STTC}_{2 \times 1}$-QPSK, URCSTTC $_{2 \times 1}$-QPSK and IrCC-URC-STTC $2 \times 1$-QPSK, as seen in Fig.6. Additionally, it can be seen from Fig.5 and Fig. 6 that the IrCC-URC-STTC-QPSK scheme's capacity curve is only about $(2.3-1.6)=0.7 \mathrm{~dB}$ away from the STTC-based DCMC capacity curve.

Table 1: Subcode weighting coeffients of the IrCC encoder associated with URC-STTC-QPSK, URCSTTC-8PSK and URC-STTC-16PSK.

\begin{tabular}{|c|c|}
\hline $\begin{array}{l}\text { Arrangement } \\
\text { (Turbo-cliff } \\
\text { SNR) }\end{array}$ & Coefficients: $\left[\alpha_{1}, \alpha_{2}, \ldots, \alpha_{17}\right]$ \\
\hline $\begin{array}{l}\text { URC-STTC- } \\
\text { QPSK }(2.3 \mathrm{~dB})\end{array}$ & 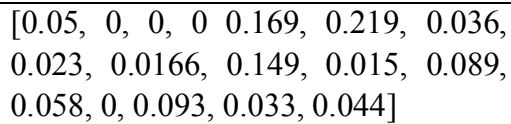 \\
\hline $\begin{array}{l}\text { URC-STTC- } \\
8 P S K(4.4 \mathrm{~dB})\end{array}$ & 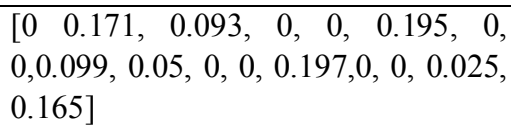 \\
\hline $\begin{array}{l}\text { URC-STTC- } \\
\text { 16PSK (7.0 dB) }\end{array}$ & $\begin{array}{l}{[0.203,0,0.093,00.102,0,0,0.148,} \\
0,0,00.055,0.149,0,0,0,0.248]\end{array}$ \\
\hline
\end{tabular}

Following the same procedure as illustrated by designing the IrCC-URC-STTC-QPSK, we also designed other coding arrangements relying on 8PSK and 16PSK, namely the IrCC-URC-STTC-8PSK and IrCC-URC-STTC-16PSK schemes. The corresponding weighting coefficients of the IrCC encoder are listed in Tab. 1.

\section{Performance of IrCC-URC-STTC-MPSK coding scheme}

Upon employing the $\mathrm{IrCC}$ weighting coefficients listed in Tab. I, we can now evaluate the BERperformance of our coding schemes, namely of IrCCURC-STTC-QPSK and IrCC-URC-STTC-16PSK, which rely on different modulation arrangements, such as QPSK, 8PSKand 16PSK, as shown in Fig.7. For the sake of clarity, it should be noted that we only present the results associated with QPSK and16PSK in Fig.7 and Fig.8. Observe in Fig.7 that our Monte-
Carlo simulation results substantiate the predictions obtained by using the EXIT-charts of Fig.5, as we illustrated earlier in Section III-B by the example of the IrCC-URC-STTC-QPSK coding scheme. For the scenarios of employing 8PSK and 16PSK, the IrCC coefficients and the corresponding 'turbo-cliff' SNRs facilitating a vanishingly low BERare summarized in Table 1, which were obtained by our EXIT-chart based design.

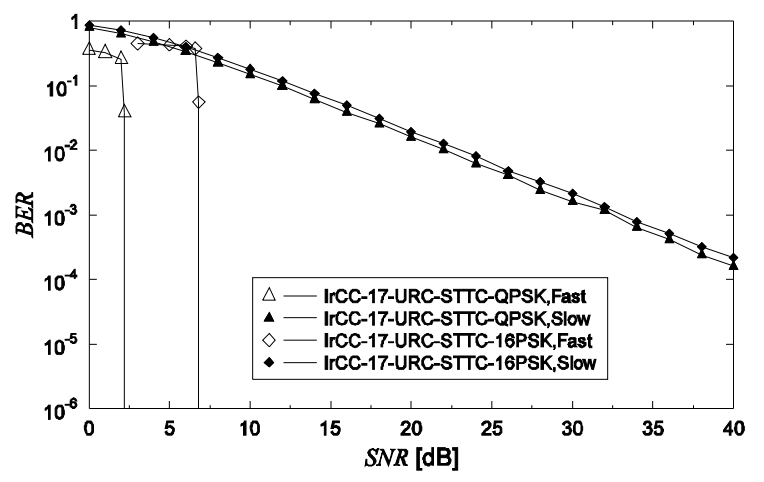

Fig. 7. BER performance of IrCC-URC-STTC2 $\times 1$ MPSK corresponding to different modulation schemes, namely QPSK, 8PSK and 16PSK over

Rayleigh small-scale (fast) and large-scale (slow) fading channels, where the coding rate $R_{c}=0.5$ and the frame length $N=10^{5}$ are employed, while the weightingcoefficients of the IrCC are given in Tab. I.

Importantly, the value of the 'turbo-cliff' SNR given in Table I indicates that as we inferred from our EXIT-chart analysis, once the SNR value exceeds this value, the BER/FER of the coding scheme is expected to become infinitesimally low. Indeed, the performance prediction provided by the EXIT-chart curves is fulfilled by our simulation results presented in Fig.7. Explicitly, the BER of the system supported by our coding schemes, such as the IrCC-URC-STTCQPSK, IrCC-URC-STTC-8PSK and IrCC-URCSTTC-16PSK arrangements, drops to a value below $10-6$, when the SNR value exceeds the corresponding turbo-cliff SNR.

However, as suggested in [38], in the small-scale fading channel, the transmitter can send data at the rate of $R<\left.C\right|_{h}$, while maintainingan arbitrarily small error probability, but this idealised performance cannot be maintained for large-scale fading channel. This phenomenonis also confirmed by the 
performance of our system employing merely the first MIMO layer in appearance of the large-scale (slow) fading in the wireless channels, as seen in Fig.7. As a result of the negative influence associated with the slow fading, our system performance experiences a degradation of about $45 \mathrm{~dB}$ at an $F E R=10^{-4}$, as seen in Fig.7. Hence, the second VMIMO layer is then proposed for eliminating the performance degradation.

\section{THE SECOND VMIMO LAYER DESIGN}

In this section, the outage probability and the associated diversity order of the VMIMO system are briefly highlighted in Sec. IV-A, in order set up bases for our design principle illustrated in Sec. IV-B.

\section{A. Outage Probability and Diversity Order}

Let direct our attention to the system model of the VMIMO layer portrayed in Fig.II. Accordingly, let $\mathrm{U}_{\mathrm{m}, \mathrm{t}}$ be a set of user indicescorresponding to the specific users that are able to correctly recover a frame $I_{m}(t)$ transmitted from User $m$ during time slot $t$. We then have $U_{m t}=\left\{u_{m t}, u_{n, t} \in 1, \ldots, M\right\}$ for $\forall \mathrm{m} \in\{1, \ldots, \mathrm{M}\}$. Note that User $\mathrm{m}$ itself is always included in this set. The number of members in theset of users $U_{m, t}$, which is denoted by $\left\|U_{m t}\right\|$, is equal to the number of users that are capable of correctly detecting the frame $I_{m}(t)$. Wedefine the complement set of $U_{m t}$ as $U_{m t}^{*}$ that comprises the indices of those users that cannot correctly recover the frame $I_{m}(t)$. Hencewe have

$$
\left\|U_{m, t}\right\|=M-\left\|U_{m, t}^{*}\right\|,
$$

Let $\mathrm{Pe}$ be the error probability of a single link corresponding to a given SNR. Assuming that all the links/users in the network employ the same channel coding scheme, the probability that the set $\mathrm{U}_{\mathrm{m}, \mathrm{t}}^{*}$ of users cannot recover the frame $\operatorname{Im}(\mathrm{t})$ is approximatelyP $\|$ the frame $I_{m}(t)$ is contained in the set of $\left(\mathrm{M}-\left\|\mathrm{U}_{\mathrm{m}, \mathrm{t}}^{*}\right\|\right) \mathrm{k} 2+$ frames, where the additional term ${ }^{\prime}+1^{\prime}$ 'represents the transmission ofUser $\mathrm{m}$ to the BS during the broadcast phase $\mathrm{m}$, while the term $\left(\mathrm{M}-|| \mathrm{U}_{\mathrm{m}, \mathrm{t}}^{*}||\right)$ k represents the number of parity frames transmitted fromall the users in the correctly decoded set $U_{m, t}$ to the BS during the cooperative phases. The outage probability representing the likelihoodof incorrectly decoding
$\mathrm{I}_{\mathrm{m}}(\mathrm{t})$ is denoted by $\mathrm{P}_{\mathrm{o}, \mathrm{m}}\left(\mathrm{U}_{\mathrm{mt}}^{*}\right)$, which is approximated as [27]

$$
P_{o, m}\left(U_{m, t}^{*}\right) \approx\left(k 1, k 2, U_{m, t}^{*}\right) P_{e}^{\left(M-\left\|U_{m, t}^{*}\right\|\right) k_{2}+1},
$$

where $\left(\mathrm{k}_{1}, \mathrm{k}, \mathrm{L}_{\mathrm{m}, \mathrm{t}}\right)$ represents the number of different outage patterns resulting in having an outage probability of $\mathrm{P}_{\mathrm{o}, \mathrm{m}} \mathrm{m}\left(\mathrm{U}_{\mathrm{m}, \mathrm{t}}^{*}\right)$.

Notably, (6) represents the outage probability associated with a specific set of $\mathrm{U}_{\mathrm{m}, \mathrm{t}}$ or $\mathrm{U}_{\mathrm{m}, \mathrm{t}}^{*}$. The outage probability $\mathrm{P}_{\mathrm{O}}$ of the entiresystem, which is also the outage probability for all possible sets $U_{m, t}^{*}$ of unsuccessfully recovering $I_{m}(t)$, may be expressed as [27]

$$
\begin{aligned}
& P_{0}=\sum_{\left\|U_{m, t}^{*}\right\|=0}^{M-1} P_{e}^{\left\|U_{m, t}^{*}\right\|}\left(1-P_{e}\right)^{M-1-\left\|U_{m, t}^{*}\right\|} P_{0, m}\left(U_{m, t}^{*}\right) \\
& \approx \sum_{\left\|U_{m, t}^{*}\right\|=0}^{M-1} P_{e}^{\left(M-\left\|U_{m, t}^{*}\right\| k_{2}+\left\|U_{m, t}^{*}\right\|+1\right.} \gamma\left(k_{1}, k_{2},\left\|U_{m, t}^{*}\right\|\right)
\end{aligned}
$$

Denoting the specific cardinality $\left\|\mathrm{U}_{\mathrm{m}, \mathrm{t}}^{*}\right\|$ that results in the lowest term in the sum of (7) by $\left\|\mathrm{U}_{\mathrm{m}, \mathrm{t}}^{*}\right\|_{\mathrm{lo}}$, and taking into account that || $\mathrm{U}_{\mathrm{m}, \mathrm{t}}^{*} \|_{\mathrm{lo}}=$ M-1 for $k_{2} \leq 2$ we have an outage probability of

$$
\begin{aligned}
& P_{0} \approx \gamma^{\prime}\left(k_{1}, k_{2},\left\|U_{m, t}^{*}\right\|_{l o}\right) P_{e}^{\left(M-\left\|U_{m, t}^{*}\right\|_{l o}\right) k_{2}+\left\|U_{m, t}^{*}\right\|_{l o}+1} \\
& =\gamma^{\prime}\left(k_{1}, k_{2}, M-1\right) P_{e}^{M+k_{2}}
\end{aligned}
$$

wherey'(k1, k2, M-1) represents the accumulated cardinalities of all cardinalities $\left\|\mathrm{U}_{\mathrm{m}, \mathrm{t}}^{*}\right\|$ for which we have $\left\|\mathrm{U}_{\mathrm{m}, \mathrm{t}}^{*}\right\|_{\mathrm{lo}_{\mathrm{o}}}=\left\|\mathrm{U}_{\mathrm{m}, \mathrm{t}}^{*}\right\|$.

Note that the outage probabilities $\mathrm{P}_{\mathrm{e}}$ appearing in Eq. (8) are calculated under the consideration of both fast and slow fading, where the fast-fading scenario would become identical to the slow fading situation, when the frame length is sufficiently long, and hence we haveE $\left[\left|h_{\mathrm{f}}\right|^{2}\right]=1$ Considering a slow fading channel, where the slow fading coefficients are constant throughout a frame, but are i.i.d random variables for the different frames, the achievable diversity order D may be defined as [38]

$$
D=\lim _{S N R \rightarrow \infty} \frac{-\log _{2} P_{o}}{\log _{2} S N R}
$$


Research, Development and Application on Information and Communication Technology

According to [27] and [29], the diversity order $\mathrm{D}_{\text {VMIMo }}$ obtained by activating the second_MIMO layer in our system may also beformulated with the aid of (8) and (9), as

$$
D_{V M M O}=M+k_{2}
$$

\section{B. Design principle of the VMIMO system}

It should be noted that the DVMIMO formula of (10) expresses the magnitude of diversity gain that obtained by activating the second VMIMO layer. Additionally, the component coding rate RVMIMO characterizing the second VMIMO layer presented in Sec.II-B may be expressed as

$$
\begin{aligned}
& R_{V M I M O} \\
& =\frac{\text { Total nu mbe of in formatior frames }}{\text { Total nu mber of transmitted frames }} \\
& =\frac{k_{1}}{k_{1}+k_{2}}
\end{aligned}
$$

By taking consideration of the diversity order $\mathrm{D}_{\text {VMIMO }}$ of Eq. (10) and the coding rate $\mathrm{R}_{\text {VMIMO }}$ of Eq. (11), it is plausible thatwe may conceive different systems having the same rate $\mathrm{R}_{\text {VMIMO }}$ but different diversity order $\mathrm{D}_{\text {VMIN }}$ by independently adjusting $\mathrm{k}_{1}$, $\mathrm{k}_{2}$ and $\mathrm{M}$. In other words, using (10) and (11), we are able to design a network-coding based system having the highest possible diversity order at a given system's rate $\mathrm{R}_{\mathrm{VMIMO}}$. A higher diversity order implies that the system is capable of achieving a higherdiversity gain in order to provide an improved performance. For notational convenience, we will characterize our system by using the set of parameters $\left(\mathrm{R}, \mathrm{M}, \mathrm{k}_{1}, \mathrm{k}_{2} ; \mathrm{G} ; \mathrm{R}_{\mathrm{VMIMO}}\right)$ and illustrate the entire system's design process with the aid of examples. Note that all operations are defined over the Galois Field $\mathrm{GF}(\mathrm{q})$, but perhaps unconventionally, we represent the GF elements with the aid of the corresponding decimal numbers for the sake of convenient readability. Let us initially consider a simple two-user system having the set of parameters $\left(\mathrm{R}=1, \mathrm{M}=2, \mathrm{k}_{1}=1 ; \mathrm{k}_{2}=\right.$

$1 ; G_{2 \times 4} ; R_{2 \times 4}=1 / 2$ ), where $G_{2 \times 4}$ was selected from the set of systematic generator matrices $\mathrm{G}$ designedfor RS codes defined over GF(4) [33], which is given by

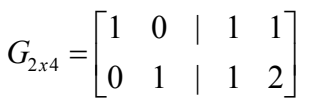

By contrast, a system defined over $\mathrm{GF}(8)$ is characterized by $\left(\mathrm{R}=1, \mathrm{M}=2, \mathrm{k}_{1}=2 ; \mathrm{k}_{2}=\right.$ 2; $\mathrm{G}_{4 \times 8} ; \mathrm{R}_{4 \times 8}=1 / 2$ ), where $\mathrm{G}_{4 \times 8}$ overGF(8) may be formulated as [33]

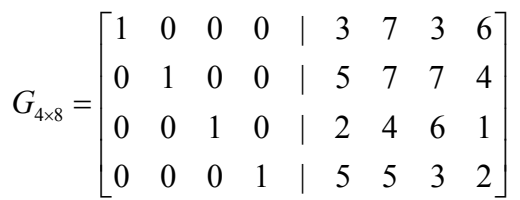

Applying both systems' parameters to (10), we can readily see that the diversity order of $D_{4 \times 8}=4$ is higher than $\mathrm{D}_{2 \times 4}=3$ while bothof their rate is $\mathrm{R}_{2 \times 4}=\mathrm{R}_{4 \times 8}=1 / 2$. This suggests that our $\mathrm{G}_{4 \times 8^{-}}$based system is capable of providing an improved FER performance incomparison to that of the $\mathrm{G}_{2 \times 4}$ based one.

\section{SIMULATION RESULTS AND DISCUSSIONS}

In this section, we will provide simulation and numerical results in order to clarify the benefit of our proposed two layer MIMO architecture in combating both slow fading and fast fading appearing in hostile wireless channels, w.r.t. our analysis presented in Sec. III and Sec. IV. For the sake of simplicity, we used the above-mentioned $G_{2 \times 4}$ and $G_{4 \times 8}$ based systems as examples for our demonstration.

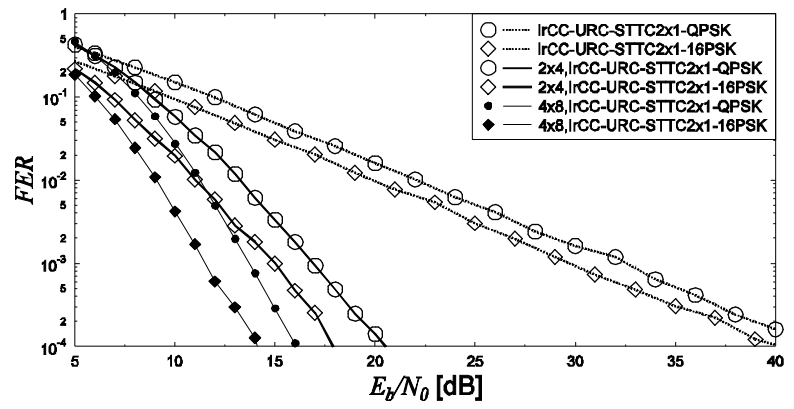

Fig. 8. FER performance of our two layer VMIMO systems employing $G_{2 \times 4}$ and $G_{4 \times 8}$ in the second VMIMO layer, where IrCC-URC-STTC2 $\times 1$-MPSKare deployed in the first MIMO layer.

It should be noted that the performance of our system in Fig.7 is presented in the form of SNRversus-FER performance, for facilitating the nearcapacity comparison between the performance results in Fig.7 and corresponding results gleaned by EXIT chart analyses and presented in Tab.I. By contrast, the performance presented in Fig. 8 is formulated in the 
form of $E_{b} / N_{0}$-versus-FER, for highlighting a fair comparison between different system configurations having distinct coding rates, which are characterized by the set of parameters mentioned in Sec.IV-B.

Let recall our results presented in Fig.7, where our system performance is shown to be degraded approximately $45 \mathrm{~dB}$ at an FER of 10-4, when the effect of the slow fading is taken into consideration, in addition to the effect of the fast fading. As mentioned in Sec. III-C, our proposed second VMIMO layer may be able to assist our system in combating the negative influence of the slow fading. As demonstrated in Fig.8, the degradation is compensated when activating the second VMIMO layer in our system. Accordingly, the compensation in our system degradation leads to an FER-performance improvement of $26 \mathrm{~dB}$ and 22 $\mathrm{dB}$ at $\mathrm{FER}=10^{-4}$, as seen in Fig.8. The improvements are obtained, when the second VMIMO layer is configured for using matrices of $\mathrm{G}_{2 \times 4}$ and $\mathrm{G}_{4 \times 8}$, respectively, provided that the IrCC-URCSTTC2 $\times 1-$ QPSK is activated in the first MIMO layer of our system.

The significant compensation in system's degradation obtained by activating the second VMIMO layer poses a subsequent question that how the network coding can assist our system in compensating the degradation caused by the slow fading channels. As analyzed in Sec.IV-B, based on formulae given in Eq. (10) and Eq. (11), the second VMIMO layer can be configured for having the same information rate but offering different diversity orders. Accordingly, we expect that a better performance is confirmed for the system associated with the higher diversity order of $\mathrm{G}_{4 \times 8}$. As seen in Fig.8, the system performance improvement of approximately $4 \mathrm{~dB}$ at an $\mathrm{FER}=10^{-4}$ may be achieved by activating the $\mathrm{G}_{4 \times 8}$ associated configuration, while $\mathrm{G}_{2 \times 4}$ associated configuration are being used in the second VMIMO layer, provided that the IrCC-URC-STTC2 $\times 1$-QPSK scheme is employed in the first MIMO layer of our system. However, what is the best compensation possible that the second VMIMO layer may support, is yet provided in this paper, we leave the issue for our further publication.

\section{CONCLUSIONS}

In this contribution, we proposed the two-layer MIMO systems using our IRCC-URC-STTC2 $\times 1$ MPSK scheme in the first MIMO layer, while the second VMIMO layer is constructed by employing network codes. Our proposed VMIMO system is proved to provide a significant performance improvement of at least $22 \mathrm{~dB}$, when comparing to the corresponding conventional MIMO systems. We also devise the design principles that may be applied in a vast range of two layer MIMO systems.

\section{REFERENCES}

[1] J. Laneman and G. Wornell, "Distributed spacetime-coded protocols for exploiting cooperative diversity in wireless networks," IEEE Transactions on Information Theory, vol. 49, pp. $2415-2425$, Oct. 2003.

[2] J. Laneman, D. Tse, and G. Wornell, "Cooperative diversity in wireless networks: Efficient protocols and outage behavior," IEEE Transactions on Information Theory, vol. 50, pp. $3062-3080$, Dec. 2004.

[3] V. Tarokh, N. Seshadri, and A. R. Calderbank, "Space-time codes for high data rate wireless communication: performance criterion and code construction", IEEE Transactions on Information Theory, vol. 44, pp. 744-765, Mar. 1998.

[4] S. M. Alamouti, "A simple transmit diversity technique for wireless communications," IEEE Journal on Selected Areas in Communications, vol. 16,pp. 1451-1458, Oct. 1998.

[5] V. Tarokh, A. Naguib, N. Seshadri, and A. R. Calderbank, "Space-time codes for high data rate wireless communication: performance criteria in the presence of channel estimation errors, mobility, and multiple paths," IEEE Transactions on Communications, vol. 47, pp. 199-207, Feb. 1999.

[6] S. X. Ng, S. Das, J. Wang, and L. Hanzo, "Nearcapacity iteratively decoded space-time block coding," in Proc. IEEE Vehicular Technology Conference VTC Spring 2008, pp. 590-594, May 11-14, 2008.

[7] L. Hanzo, O.Alamri, M. E.Hajjar and N.Wu, NearCapacity Muti-Functional MIMO Systems. New York, USA : John Wiley and Sons, 2009.

[8] M.Tuchler and J. Hagenauer, "Exit charts of irregular codes," in Proceeding of the 36th Annual Conference on Information Sciences and Systems[CDROM], (Princeton, NJ, USA), March 2002.

[9] M. Tuchler, "Design of serially concatenated systems depending on the block length," IEEE 
Research, Development and Application on Information and Communication Technology

Transactions on Communications, vol. 52, pp. 209218,Feb. 2004.

[10] S. ten Brink, "Convergence behavior of iteratively decoded parallel concatenated codes," IEEE Transactions on Communications, vol. 49, pp. 17271737,Oct. 2001.

[11] M. Tuchler, "Convergence prediction for iterative decoding of threefold concatenated systems," in Proc. IEEE Global Telecommunications Conference GLOBECOM '02, vol. 2, pp. 1358-1362, Nov. 1721, 2002.

[12] S. X. Ng, J. Wang, M. Tao, L.-L. Yang, and L. Hanzo, "Iteratively decoded variable length spacetime coded modulation: Code construction and convergence analysis," IEEE Transactions on Wireless Communications, vol. 6, pp. 1953-1963, May 2007.

[13] R. Tee, R. G. Maunder, and L. Hanzo, "Exit-chart aided near-capacity irregular bit-interleaved coded modulation design," IEEE Transactions on Wireless Communications, vol. 8, pp. 32-37, Jan. 2009.

[14] Y. Li and B. Vucetic, "On the performance of a simple adaptive relaying protocol for wireless relay networks," in Proc. IEEE Vehicular Technology Conference VTC Spring 2008, pp. 2400 -2405, May 2008.

[15] T. Hunter and A. Nosratinia, "Diversity through coded cooperation," IEEE Transactions on Wireless Communications, vol. 5, pp. 283 - 289, Feb. 2006.

[16] R. Ahlswede, N. Cai, S.-Y. Li, and R. Yeung, "Network information flow," IEEE Transactions on Information Theory, vol. 46, pp. $1204-1216$, July2000.

[17] A. Asterjadhi, E. Fasolo, M. Rossi, J. Widmer, and M. Zorzi, "Toward network coding-based protocols for data broadcasting in wireless ad hoc networks", IEEE Transactions on Wireless Communications, vol. 9, pp. $662-673$, Feb. 2010.

[18] C. Fragouli and E. Soljanin, "Network coding fundamentals," Foundation and Trends in Networking, vol. 2, no. 1, pp. 1-133, 2007.

[19] Z. Zhang, "Theory and applications of network error correction coding," Proceedings of the IEEE, vol. 99, pp. 406 -420, Mar. 2011.

[20] Y. R.W., C. N. Li S.-Y.R., and Z. Z., "Network coding theory," Network Coding Theory, Foundations and Trends in Communication and Information Theory, vol. 2, no. 4 and 5, pp. 241381, 2006.

[21] D. Traskov, M. Heindlmaier, M. Medard, and R. Koetter, "Scheduling for network-coded multicast," Networking, IEEE/ACM Transactions on, vol. 20,pp. $1479-1488$, Oct. 2012.

[22] B. Li and Y. Wu, "Network coding [scanning the issue]," Proceedings of the IEEE, vol. 99, pp. $363-$ 365, Mar. 2011.
[23] S.-Y. Li, Q. Sun, and Z. Shao, "Linear network coding: Theory and algorithms," Proceedings of the IEEE, vol. 99, pp. 372 -387, Mar. 2011.

[24] R. Koetter and M. Medard, "An algebraic approach to network coding," IEEE/ACM Transactions on Networking, vol. 11, pp. 782 - 795, Oct. 2003.

[25] T. Ho, M. Medard, R. Koetter, D. Karger, M. Effros, J. Shi, and B. Leong, "A random linear network coding approach to multicast," IEEE Transactions on Information Theory, vol. 52, pp. $4413-4430$, Oct. 2006.

[26] M. Jafari, L. Keller, C. Fragouli, and K. Argyraki, "Compressed network coding vectors," in 2009 IEEE International Symposium on Information Theory (ISIT 2009), pp. 109 -113, June-July 2009.

[27] J. L. Rebelatto, B. F. Uchôa-Filho, Y. Li, and B. Vucetic, "Generalized distributed network coding based on nonbinary linear block codes for multi-user cooperative communications," in 2010 IEEE International Symposium on Information Theory (ISIT 2010), pp. 943 -947, June 2010.

[28] M. Xiao and M. Skoglund, "M-user cooperative wireless communications based on nonbinary network codes," in IEEE Information Theory Workshop on Networking and Information Theory, 2009. (ITW 2009), pp. 316 -320, June 2009.

[29] J. Rebelatto, B. Uchoa-Filho, Y. Li, and B. Vucetic, "Multiuser cooperative diversity through network coding based on classical coding theory," IEEE Transactions on Signal Processing, vol. 60, pp. 916926, Feb 2012.

[30] H. V. Nguyen, C. Xu, S. X. Ng, and L. Hanzo, "Non-coherent near-capacity network coding for cooperative multi-user communications," IEEE Transactions on Communications, vol. 60, pp. 30593070, October 2012.

[31] H. V. Nguyen, S. X. Ng, and L. Hanzo, "Irregular convolution and unity-rate coded network-coding for cooperative multi-user communications," IEEE Transactions on Wireless Communications, vol. 12, pp. 1231-1243, March 2013.

[32] C. Hausl and P. Dupraz, "Joint Network-Channel Coding for the Multiple-Access Relay Channel," in 3rd Annual IEEE Communications Society on Sensor and Ad Hoc Communications and Networks, 2006. SECON '06, vol. 3, pp. 817 -822, Sept. 2006.

[33] SAGE, "Open source mathematics software," in online source available at http://wwwsagemath.org/.

[34] S. X. Ng and L. Hanzo, "On the MIMO channel capacity of multidimensional signal sets," IEEE Transactions on Vehicular Technology, vol. 55, pp. 528-536, March 2006.

[35] S. X. Ng, J. Wang, and L. Hanzo, "Unveiling NearCapacity Code Design: The Realization of Shannon's Communication Theory for MIMO Channels", in Proc. IEEE International Conference 
on Communications ICC '08, pp. 1415-1419, May 19-23, 2008.

[36] A. Ashikhmin, G. Kramer, and S. ten Brink, "Extrinsic information transfer functions: model and erasure channel properties," IEEE Transactions on Information Theory, vol. 50, pp. 2657-2673, Nov. 2004.

[37] S. ten Brink, "Rate one-half code for approaching the Shannon limit by $0.1 \mathrm{~dB}$," Electronics Letters, vol. 36, pp. 1293-1294, July 20, 2000.

[38] D. Tse and P. Viswanath, Fundamentals of Wireless Communications, Englewood Cliffs, NJ, USA: Cambridge: Cambridge University Press, 2005.

\section{AUTHORS' BIOGRAPHIES}

Dr. Hung Viet Nguyen obtained a $\mathrm{PhD}$ in Electronics and Electrical Engineering from School of Electronics \& Computer Science, University of Southampton, UK in 2013. Previously, he received a B.Eng. in Electronics \& Telecommunications from Hanoi University of Technology and MEng. in Telecommunications from Asian Institute of Technology, in 1999 and 2002, respectively. His research interests focus on Cooperative Communications, Channel Coding, Network Coding \& Cognitive Communications. His further details may be found upon following the url as https://sites.google.com/site/nguyenviethungngvihu/

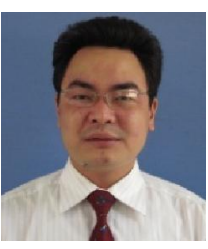

Dam Viet Nguyen received a MSc in Post and Telecommunication Institute of Technology (PTIT) in 2010. In the present, he has been a lecturer at PTIT. His research interests focus on Simulation of Communication Systems, Mobile Fading Channels, Resource Allocation in Wireless Communication Systems.

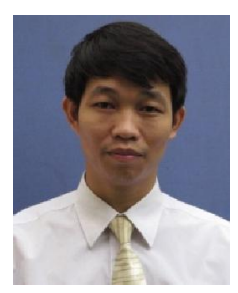

Dr. Sy Quy Nguyen received his B.Sc in 1995 from Hanoi University of Technology, Vietnam and his Ph.D. degree of telecommunication engineering from Post and Telecommunication Institute of Technology (PTIT) in 2003. From 6/1995 to 2004, he was a researcher at the Switching Department, Research Institute of Posts and Telecom (RIPT). From 2004 to present, he has been a lecturer at
PTIT. His research interests are in the fields of switching system, access networks, data communication, advanced computer architectures, and wireless networks. 